\title{
Stable Isotope Labeling Strategy for Curcumin Metabolite Study in Human Liver Microsomes by Liquid Chromatography-Tandem Mass Spectrometry
}

\author{
Dan Gao, ${ }^{1,2}$ Xiaowu Chen, ${ }^{1,2}$ Xiaomei Yang, ${ }^{5}$ Qin Wu, ${ }^{1,2}$ Feng Jin, ${ }^{3}$ Hongliang Wen, ${ }^{5}$ \\ Yuyang Jiang, ${ }^{1,4}$ Hongxia Liu ${ }^{1,2}$
}

${ }^{1}$ State Key Laboratory Breeding Base-Shenzhen Key Laboratory of Chemical Biology, Graduate School at Shenzhen, Tsinghua University, Shenzhen, 518055, China

${ }^{2}$ Key Laboratory of Metabolomics at Shenzhen, Shenzhen, 518055, China

${ }^{3}$ Neptunus Pharmaceutical Technology Center, Shenzhen, 518057, China

${ }^{4}$ School of Medicine, Tsinghua University, Beijing, 100084, China

${ }^{5}$ School of Chemical Engineering and Environment, Beijing Institute of Technology, Beijing, 100081, China

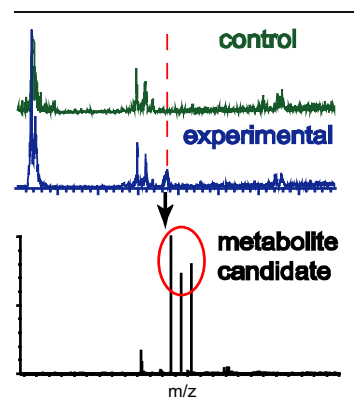

\begin{abstract}
The identification of drug metabolites is very important in drug development. Nowadays, the most widely used methods are isotopes and mass spectrometry. However, the commercial isotopic labeled reagents are usually very expensive, and the rapid and convenient identification of metabolites is still difficult. In this paper, an ${ }^{18} \mathrm{O}$ isotope labeling strategy was developed and the isotopes were used as a tool to identify drug metabolites using mass spectrometry. Curcumin was selected as a model drug to evaluate the established method, and the ${ }^{18} \mathrm{O}$ labeled curcumin was successfully synthesized. The non-labeled and ${ }^{18} \mathrm{O}$ labeled curcumin were simultaneously metabolized in human liver microsomes (HLMs) and analyzed by liquid chromatography/mass spectrometry (LC-MS). The two groups of chromatograms
\end{abstract} obtained from metabolic reaction mixture with and without cofactors were compared and analyzed using Metabolynx software (Waters Corp., Milford, MA, USA). The mass spectra of the newly appearing chromatographic peaks in the experimental sample were further analyzed to find the metabolite candidates. Their chemical structures were confirmed by tandem mass spectrometry. Three metabolites, including two reduction products and a glucuronide conjugate, were successfully detected under their specific HLMs metabolic conditions, which were in accordance with the literature reported results. The results demonstrated that the developed isotope labeling method, together with post-acquisition data processing using Metabolynx software, could be used for fast identification of new drug metabolites.

Keywords: Curcumin, ${ }^{18} \mathrm{O}$ isotope labeling, Metabolite, HLMs, HPLC/QqQ-MS

Received: 27 September 2014/Revised: 18 November 2014/Accepted: 9 December 2014/Published Online: 16 January 2015

\section{Introduction}

$\mathrm{T}$ he investigation of drug metabolites in vitro and in vivo is one of the essential studies in the early stages of drug development [1]. Meanwhile, more drug safety evaluation is needed because metabolites generated from phase I biotransformations are more likely to be chemical reactive, pharmacologically active, or toxic. However, the identification of drug metabolites is very difficult because the analytical signals

Correspondence to: Hongliang Wen; e-mail: wen.hongliang816@gmail.com, Hongxia Liu; e-mail: liuhx@sz.tsinghua.edu.cn derived from drug metabolites are weaker than the signals from complex matrix [2]. Isotopic tracers in which a specific atom or atoms replaced with a different isotope have been used as a key technique to analyze the metabolic pathway [3]. The isotopic tracers can be either radioactive or stable; however, the major disadvantage of radiolabeling is the risk of exposure in humans. Therefore, stable isotope labeling has become the mostly widely used method for the structural identification of metabolites in drug discovery, especially for new drugs [4]. Stable isotopes such as carbon-13 [5, 6], nitrogen-15 [7, 8], and deuterium [912] could be incorporated strategically into drug molecules to assist the structure elucidation of their metabolites using LC/ MS and NMR. Numerous studies have reported using the 
mixtures of equal amount of non-labeled and isotope-labeled compounds to study in vitro and in vivo metabolic disposition and compounds [13-15]. Because the labeled and unlabeled analogues retained almost identical physicochemical properties, the separation of the parent compound or its metabolites to form a twin ion pair pattern by mass spectrometry could help detect and identify the unknown drug metabolites. Moreover, the incorporation of stable isotopes into the parent compound also provides an opportunity to explore the possible metabolic pathways or the enzymes responsible for the formation of the metabolites. Another example is the use of oxygen-18 labeled oxygen molecules $\left({ }^{18} \mathrm{O}_{2}\right)$ and water $\left(\mathrm{H}_{2}{ }^{18} \mathrm{O}\right)$ in metabolism studies to understand the metabolic pathways or metabolites structures [16, 17]. However, the incorporation of oxygen-18 into the compounds for drug metabolism research has not been reported. Currently, liquid chromatography-tandem mass spectrometry (LC-MS/MS) has become an important and reliable analytical technique for metabolites identification because of its high separation ability and the ability to provide structural information. Furthermore, tandem mass spectrometry (MS/ MS) can be used to confirm chemical structures of metabolites through their mass-to-charge ratio $(\mathrm{m} / \mathrm{z})$ and MS/MS fragmentations with high resolution [18].

Curcumin, [1,7-bis(4-hydroxy-3-methoxyphenyl)-1,6heptadiene-3,5-dione], is a potent antioxidant extracted from the powered rhizomes of turmeric (Curcuma longa). Turmeric is widely used as a food coloring and flavoring agent. Many studies have shown that curcumin possessed a wide range of biological and pharmacological activities including anti-inflammatory, anti-viral, anti-oxidant, anti-carcinogenic, and cancer chemoprevention activities [19-23]. The studies have indicated that it has no serious toxicity on animal models or humans even at very high doses. Its wonderful biological activities and pharmacological safety make it as a potential therapeutic reagent for the treatment of several human diseases $[24,25]$. However, there are several inherent drawbacks that prevent its entering the market as a drug so far. The major obstacles are the poor aqueous solubility and absorption, rapid metabolism, chemical instability, and low oral bioavailability [26-28]. In addition, several researches have implied that curcumin metabolites might regulate some of the biological effects of curcumin [29-31]. The metabolic pathways have been widely studied. In vivo and in vitro studies have demonstrated that curcumin undergoes both phase I and phase II metabolism [32-34]. Phase I biotransformation of curcumin generates serial reduction products by reduction of the olefinic double bonds, including tetrahydro-curcumin, hexahydrocurcumin, and octahydro-curcumin. Phase II metabolism of curcumin is the conjunction of curcumin and its reductive metabolites with glucuronic and sulfate. Glucuronidation is the major phase II conjugation reaction of curcumin in humans [35]. Therefore, reduction and conjugation are regarded as the common metabolic pathways of curcumin that take place in hepatic and intestinal tissues [32, 36]. In mammals, the liver is the major organ involved in the metabolism of various endogenous compounds and drugs. The cytochrome P450 (CYP) superfamily is a large and diverse group of enzymes that is involved in drug metabolism and bioactivation, and $75 \%$ of the total number of different metabolic reactions are catalyzed by them [37]. Human liver microsomes (HLMs) are commonly used as the carrier of P450 and have been widely used for in vitro drug metabolism studies because of advantages including low cost, simplicity of use, and fewer ethical objections $[38,39]$.

Because of the importance of a drug metabolism study, we developed an ${ }^{18} \mathrm{O}$ isotope labeling strategy to analyze the metabolites of curcumin in HLMs by LC-MS with Metabolynx analyzer (Waters Corp., Milford, MA, USA). Owing to the clear metabolic pathways of curcumin, it was selected as a model drug to evaluate the reliability of the established method. The ${ }^{18} \mathrm{O}$ labeled curcumin was synthesized, and the reaction products mixture, including curcumin, $\left[{ }^{18} \mathrm{O}\right]$-curcumin, and $\left[{ }^{18} \mathrm{O}_{2}\right]$-curcumin were then simultaneously metabolized by HLMs - three major metabolites including two reduction products and a glucuronide conjugate, in accordance with the literature reported results. Our developed method with the advantages of minimal operator intervention and reduced data interpretation time is expected to be applied for the metabolism study of other compounds by the stable isotope labeling strategy.

\section{Experimental}

\section{Chemicals and Reagents}

$\mathrm{H}_{2}{ }^{18} \mathrm{O}$ was purchased from Shanghai Engineering Research Center of Stable Isotope (Shanghai, China). $\mathrm{K}^{18} \mathrm{OH}$ was prepared by the reaction of $\mathrm{H}_{2}{ }^{18} \mathrm{O}$ with t-BuOK in dry tetrahydrofuran and dried under vacuum before use. All other chemicals were of analytical grade. All solvents used in this study were HPLC-grade. Acetonitrile was obtained from Merck (Darmstadt, Germany). Uridine 5'-diphosphoglucuronic acid (UDPGA), nicotinamide adenine dinucleotide phosphate (NADPH), D-saccharic acid-1,4-lactone, HLMs, magnesium chloride $\left(\mathrm{MgCl}_{2}\right)$, and ammonium acetate were purchased from Sigma-Aldrich Chemical Co. (St. Louis, MO, USA).

\section{Synthesis of ${ }^{18} \mathrm{O}$ Labeled Curcumin}

As shown in Scheme 1, $\left[{ }^{18} \mathrm{O}\right]-2$-methoxy-4-methylphenol was obtained via hydroxylation of 1-iodo-2-methoxy-4-methylbenzene with $\mathrm{K}^{18} \mathrm{OH}$ in $t$-BuOH-DMSO- $\mathrm{H}_{2}{ }^{18} \mathrm{O}$ catalyzed with $\mathrm{CuI} / 8$-hydroxyquinoline. The oxidation of the methyl group at $\mathrm{C}-4$ of $\left[{ }^{18} \mathrm{O}\right]-2$-methoxy-4-methylphenol with 2,3-dichloro5,6-dicyano-1,4-benzoquinone (DDQ) in $\mathrm{CH}_{2} \mathrm{Cl}_{2}$ at room temperature gave $\left[{ }^{18} \mathrm{O}\right]$-vanillin. Finally, condensation of vanillin and $\left[{ }^{18} \mathrm{O}\right]$-vanillin with pentane-2,4-dione gave curcumin, $\left[{ }^{18} \mathrm{O}\right]$-curcumin and $\left[{ }^{18} \mathrm{O}_{2}\right]$-curcumin.

\section{Incubations with HLMs for Curcumin Metabolism}

Metabolites of curcumin were generated by incubation with HLMs in the presence of appropriate cofactors according to the 


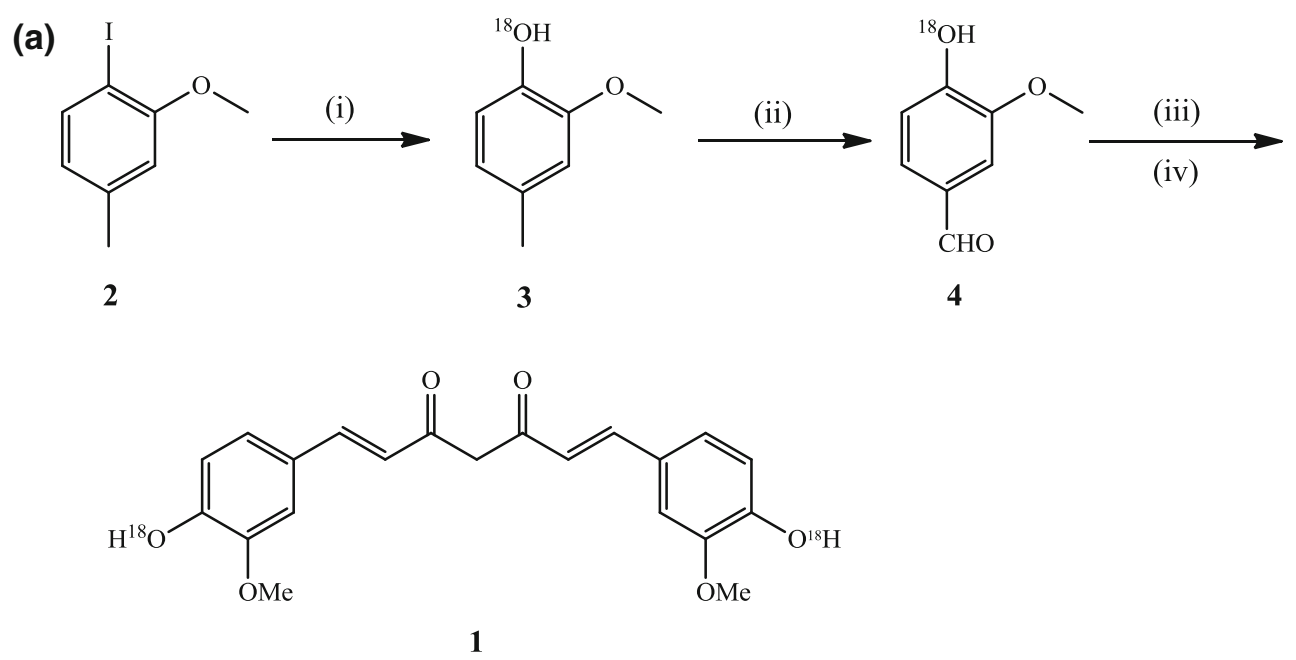

Scheme 1. Reagents and conditions: (i) Cul, 8-hydroxyquinoline, $\mathrm{K}^{18} \mathrm{OH}, t-\mathrm{BuOH}-\mathrm{DMSO}-\mathrm{H}_{2}{ }^{18} \mathrm{O}, 100{ }^{\circ} \mathrm{C}, 48 \mathrm{~h}, 87.5 \%$; (ii) $\mathrm{DDQ}$, $\mathrm{CH}_{2} \mathrm{Cl}_{2}$, rt, $4 \mathrm{~h}, 75.2 \%$; (iii) pentane-2, 4-dione, $\mathrm{B}_{2} \mathrm{O}_{3}, 1,2,3$, 4-tetrahedroquinoline, acetic acid, $110^{\circ} \mathrm{C}, 24 \mathrm{~h}$; (iv) aq acetic acid (20\%), $20^{\circ} \mathrm{C}, 24 \mathrm{~h}, 80.5 \%$ (two steps)

reported procedures [40]. Briefly, HLMs were pre-incubated on ice for $30 \mathrm{~min}$. Curcumin and ${ }^{18} \mathrm{O}$ isotope-labeled curcumin dissolved in DMSO with the final concentration of $20 \mu \mathrm{g}, 10$ $\mathrm{mM} \mathrm{MgCl}_{2}$, and $0.15 \mathrm{mg} \mathrm{HLMs}$ were added to $100 \mathrm{mM}$ phosphate buffer solution ( $\mathrm{pH} 7.4)$ to incubate for $5 \mathrm{~min}$ at $37^{\circ} \mathrm{C}$. Subsequently, NADPH with the final concentration of 1 $\mathrm{mM}$ was added to start the reactions and incubated at $37^{\circ} \mathrm{C}$ in shaking incubator block to generate reduction metabolites. After $90 \mathrm{~min}$, reactions were terminated by adding $150 \mu \mathrm{L}$ of ice-cold acetonitrile. For glucuronidation of curcumin by HLMs, $20 \mu \mathrm{g}$ curcumin, and O-18 isotope-labeled curcumin dissolved in DMSO, $0.15 \mathrm{mg}$ HLMs in $100 \mathrm{mM}$ phosphate buffer solution ( $\mathrm{pH}$ 7.4), $10 \mathrm{mM} \mathrm{MgCl} 2,10 \mathrm{mM}$ D-saccharic acid 1,4-lactone, and $2 \mathrm{mM}$ UDPGA were incubated at $37^{\circ} \mathrm{C}$ for $1.5 \mathrm{~h}$. Control incubations were conducted with HLMs in the absence of NADPH or UDPGA. All incubations were carried out in triplicate. Samples were vortexed and centrifuged at $10,000 \mathrm{~g}$ for $5 \mathrm{~min}$ at $4^{\circ} \mathrm{C}$. The supernatants were filtered and analyzed by HPLC-MS.

\section{LC-MS Analysis}

LC/MS analysis was performed on a system equipped with an Alliance HPLC Waters 2695 system (Waters) and a Quattro Premier XE mass spectrometer (Waters). The HPLC system was comprised of a quaternary pump, an online degasser, a column heater, and an autosampler. HPLC separation of curcumin and its metabolites was carried out on a X Bridge C8 column $(2.1 \mathrm{~mm} \times 100 \mathrm{~mm}$ i.d., $3.5 \mu \mathrm{m}$, Waters $)$ with the following solvent system: $\mathrm{A}=5 \mathrm{mM}$ ammonium in water, $\mathrm{B}=$ $5 \mathrm{mM}$ ammonium in acetonitrile in negative ion mode at a flow rate of $0.3 \mathrm{~mL} \mathrm{~min}^{-1}$. Elution gradient was linearly increased from $10 \%$ to $90 \%$ B within $30 \mathrm{~min}$, followed by return to $10 \%$ B. Total running time was $35 \mathrm{~min}$ per separation. Column temperature was set at $25^{\circ} \mathrm{C}$ and the injection volume was $10 \mu \mathrm{L}$.

The MS system consisted of an ESI interface and a triple quadrupole (QqQ) mass analyzer. The MS parameters were as follows: capillary voltage was set at $3.0 \mathrm{kV}$ and cone voltage was maintained at $25 \mathrm{~V}$ in negative ion mode. Source temperature was kept at $120^{\circ} \mathrm{C}$ whereas the desolvation temperature was $250^{\circ} \mathrm{C} . \mathrm{N}_{2}$ was used for desolvation gas with the flow rate of $500 \mathrm{~L} \mathrm{~h}^{-1}$. For MS/ MS analysis, the collision energy was set at $15 \mathrm{eV}$ whereas the flow rate of the collision gas was $0.15 \mathrm{~mL} \mathrm{~min}{ }^{-1}$. The samples were analyzed in full-scan and selected ion recording (SIR) mode. All data collected in centroid mode were processed using a Waters Metabolynx (v 4.1) program.

\section{Data Analysis and Metabolite Identification}

The chromatograms of the in vitro metabolic reaction mixture from the non-labeled and the ${ }^{18} \mathrm{O}$ isotopelabeled curcumin with or without cofactors were collected by separate LC-MS measurements. The reaction mixture without addition of cofactors was regarded as control. By comparison of the chromatograms corresponding to experimental and control samples, several new chromatographic peaks could be easily found in the chromatogram of the experimental sample using Metabolynx software. Metabolynx is a post-acquisition data processing software that employs an extensive list of potential biotransformation reactions consisting of hydrogenation and glucuronidation, in combination with the elemental compositions of the substrate molecules, to generate a series of extracted ion chromatograms (EICs). These EICs are compared between the control and experimental sample to eliminate those chromatographic peaks in the 
sample that also appear in the control. Only those peaks with the area ratio of experimental to control sample above 5 were considered to be the compound-related metabolites. The mass spectra of these chromatographic peaks should be further analyzed to validate whether it was the compound-related metabolites. Finally, the chemical structures of the metabolite candidates were further confirmed by tandem mass spectrometry (MS/MS).

\section{Results and Discussion}

\section{Characterization of the Synthesized \\ ${ }^{18}$ O Isotope-Labeled Curcumin}

${ }^{18} \mathrm{O}$ isotope-labeled curcumin were synthesized according to the procedures described in Scheme 1. The chemical structures of synthesized labeled and unlabeled curcumin were characterized by ESI-QqQ-MS/MS. Mass spectrometry results were obtained in the mass range $m / z 50-1000$ in the negative ion mode. As shown in Figure 1, curcumin with $[\mathrm{M}-\mathrm{H}]^{-}=$ 366.94, $\left[{ }^{18} \mathrm{O}\right]$-curcumin with $[\mathrm{M}-\mathrm{H}]^{-}=368.92$, and $\left[{ }^{18} \mathrm{O}_{2}\right]-$ curcumin with $[\mathrm{M}-\mathrm{H}]^{-}=370.94$ could be detected. The ratio of their relative mass signal intensities was about $2: 1: 1$. The result indicated that the amounts of the reaction-generated $\left[{ }^{18} \mathrm{O}\right]$-curcumin and $\left[{ }^{18} \mathrm{O}_{2}\right]$-curcumin were nearly the same because of the similar chemical properties and similar ionization capacity.

\section{Strategy for Metabolite Discovery and the Identification of Its Metabolites}

The detection of drug metabolites is very important but it is very difficult because most of the metabolites in real biological samples are at very low concentrations. In addition, the interference signals from the background will also be serious. Therefore, it requires a higher sen-

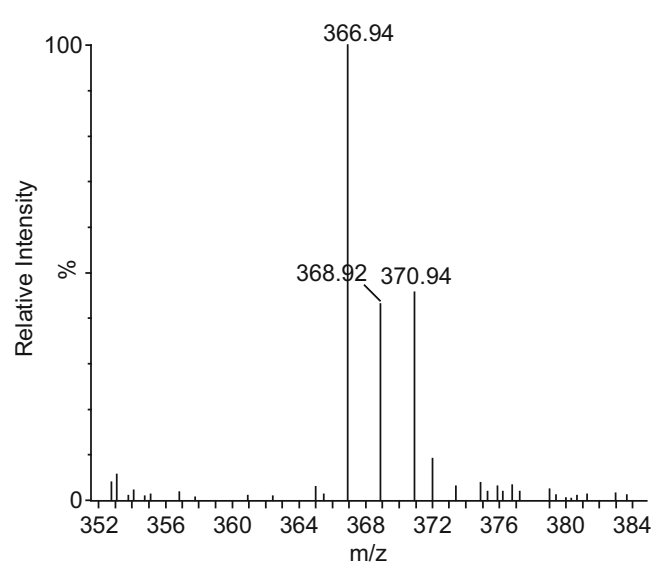

Figure 1. Mass spectrum of curcumin and ${ }^{18} \mathrm{O}$ isotope labeled curcumin. Spectrum was obtained in the negative ion mode sitivity detection method and new strategies to solve these problems. In this study, an ${ }^{18} \mathrm{O}$ isotope labeling strategy was applied for the discovery of metabolite candidates. ${ }^{18} \mathrm{O}$ isotope-labeled curcumin was synthesized and was used as a model to evaluate the feasibility of the method. Briefly, $20 \mu \mathrm{M}$ of non-labeled and ${ }^{18} \mathrm{O}$ isotope-labeled curcumin mixture were incubated in HLMs with and without cofactors (corresponding to experimental sample and control sample), and the reaction mixtures were then analyzed by LC-MS. The discovery of metabolite candidates is to compare the chromatograms corresponding to experimental and control samples using Metabolynx software and to find those chromatographic peaks only present in the experimental sample. Only those peaks with the area ratio of experimental to control sample above 5 were considered to be the potential metabolites of curcumin. The representative chromatographic results of the two groups obtained from the experimental group and control group about the glucuronidation and reduction system are shown in Figure 2. During the data processing using Metabolynx software, a large number of peaks were generated because of the existence of unrelated peaks. Therefore, we must pick up some peaks that are likely to be curcuminrelated metabolites. For glucuronidation of curcumin, four additional chromatographic peaks with high signal intensity were found present in the experimental sample, which are indicated by vertical red lines in Figure 2a and b. Therefore, they were selected for further analysis to find the potential glucuronide metabolites. Taking the results from the Metabolynx software together with the MS data containing three $\mathrm{m} / \mathrm{z}$ values with specific massshift of $2.00 \pm 0.02 \mathrm{Da}$, it was indicated that only the mass spectrum of the chromatographic peak at the retention time of 9.28 had the matched mass-shift, which was suspected to the glucuronide metabolite, and the three ions had the $\mathrm{m} / \mathrm{z}$ values of $542.78,544.77$, and 546.79, respectively, as shown in Figure 3. Besides, the relative mass spectrometry signal intensities of the three ions were similar with the labeled and non-labeled parent compounds. From the corresponding $\mathrm{m} / \mathrm{z}$ values obtained from the glucuronidation reaction system, we deduced that the most probable metabolite was the glucuronide conjugate of curcumin. The results of the curcumin reduction in HLMs are shown in Figure $2 \mathrm{c}$ and d. From the results, we could see that several chromatographic peaks were found at the retention time of $16.65 \mathrm{~min}$ and from 24.71 to $30.69 \mathrm{~min}$ in the experimental sample, but there were no three paired ions with the mass difference of $2.00 \pm 0.02 \mathrm{Da}$ from the retention time of 24.71 to $30.69 \mathrm{~min}$. However, the mass spectrum of the chromatographic peak at the retention time of $16.65 \mathrm{~min}$ had several groups of ions with $\mathrm{m} / z$ values of 367.05 , $369.03,371.08,373.04$, and 375.06 , as shown in the 

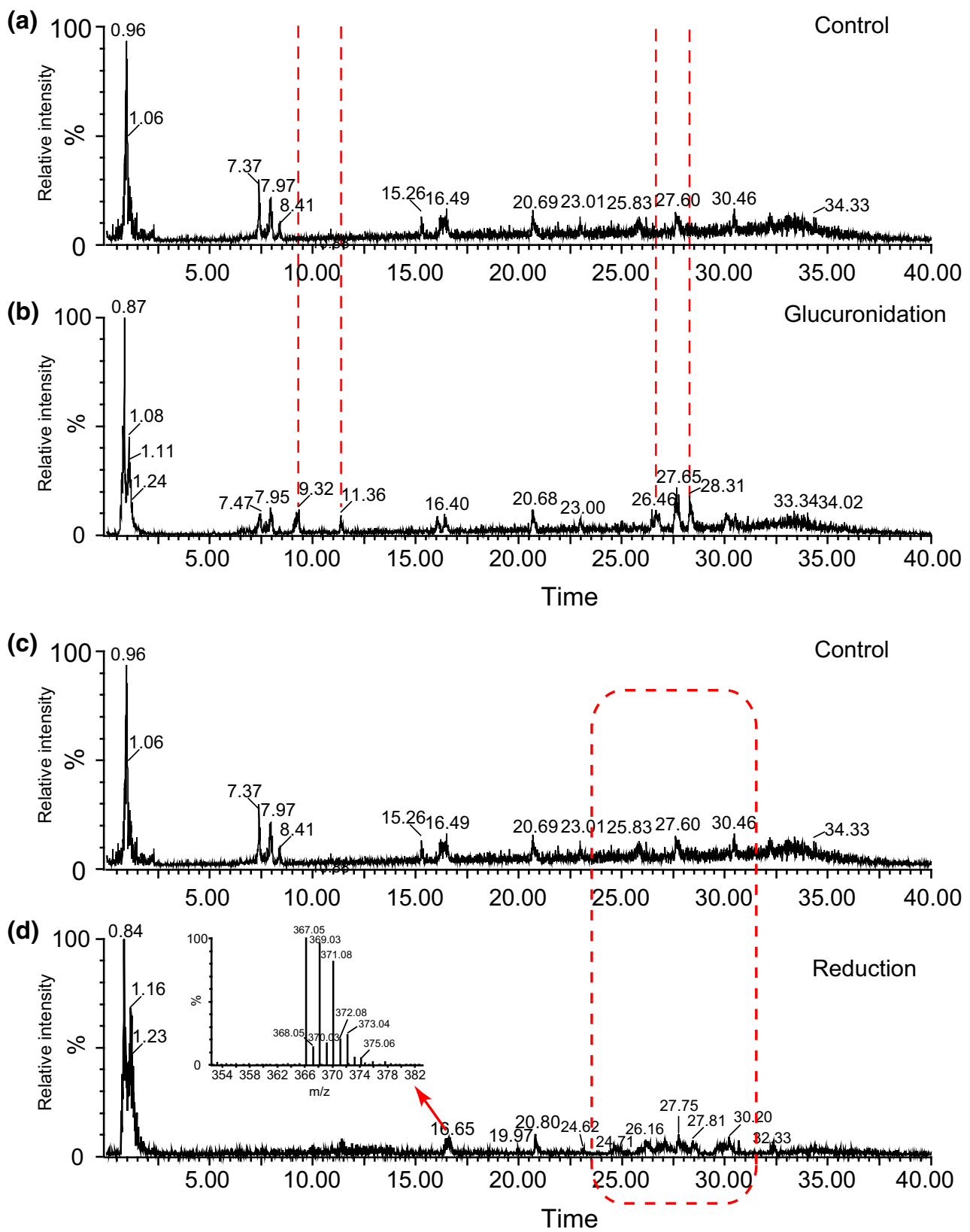

Figure 2. Analytical ion chromatograms of the mixtures of curcumin and ${ }^{18} \mathrm{O}$ isotope labeled curcumin metabolized in HLMs obtained in the negative ion mode. The metabolic reaction mixture without addition of cofactors was as control. (a, b) Glucuronidation of the curcumin mixture; (c, d) reduction of the curcumin mixture. The insert mass spectrum was obtained from the chromatographic peak at the retention time of $9.28 \mathrm{~min}$. The vertical red lines or rectangle indicate the different chromatographic peaks between the experimental and control samples

inset mass spectrum of Figure $2 \mathrm{~b}$. These ions could match with the mass-shift of $2.00 \pm 0.02 \mathrm{Da}$, but they could not be confirmed as the reduction metabolites because of the same molecular weight between reduction metabolites and O-18-labeled curcumin. For example, $\left[{ }^{18} \mathrm{O}\right]$-curcumin and dihydro-curcumin, $\left[{ }^{18} \mathrm{O}_{2}\right]$-curcumin and tetrahydro-curcumin, dihydro- $\left[{ }^{18} \mathrm{O}_{2}\right]$-curcumin and tetrahydro- $\left[{ }^{18} \mathrm{O}\right]$-curcumin have the same molecular weight. The major reason might be their overlapped chromatographic peaks between the curcumin and its reduction metabolites because of their similar chemical structures and properties. Besides, the concentration of 


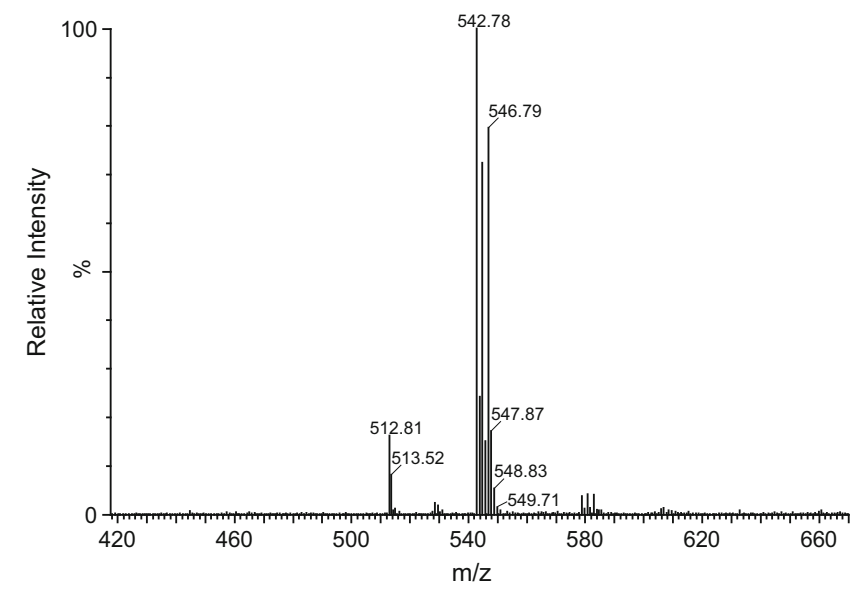

Figure 3. The corresponding mass spectrum of the chromatographic peak at the retention time of $9.28 \mathrm{~min}$. The spectrum was obtained in the negative ion mode

reduction metabolites of curcumin might be at a very low level. Therefore, a new analytical strategy or new separation condition should be adopted to further confirm the above detection result.

A new separation condition and a new data acquisition mode were applied in the following experiment. The optimized elution gradient condition was that A was linearly decreased from $80 \%$ to $40 \%$ within $15 \mathrm{~min}$. It was well known that selected ion recording (SIR) mode had the advantage of enhanced sensitivity and reduced measurement time for the monitored ions. In order to provide confirmation at the higher resolution and to determine if identification was possible at the lower resolution, mass spectra acquisition was made in SIR mode, with a dwell time of $100 \mathrm{~ms}$. Several researches have shown that dihydro-curcumin, tetrahydro-curcumin, and

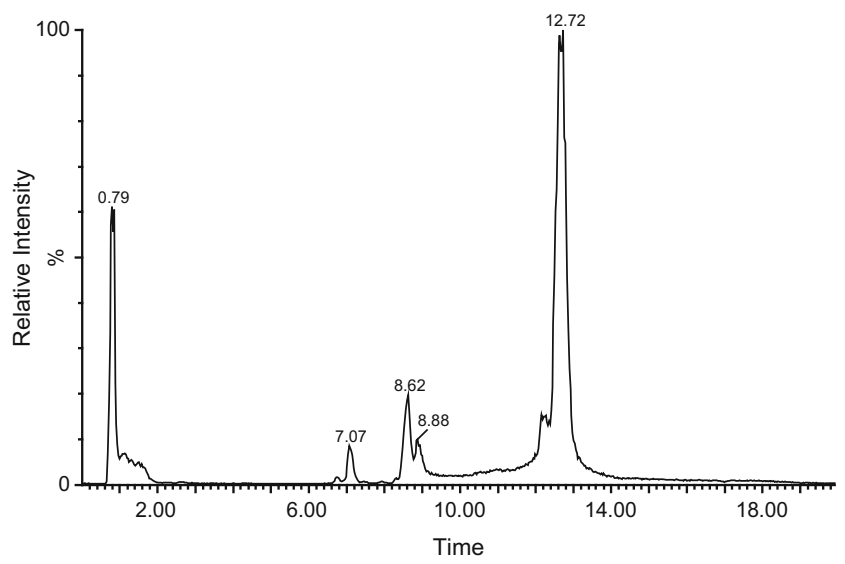

Figure 4. A typical SIR chromatogram of four monitored ions at $m / z 367,369,371$, and 375 in curcumin reduction system hexahydro-curcumin were the major products among the reduction products of curcumin. Of those, tetrahydro-curcumin was one of the major metabolites to exhibit the same physiological and pharmacologic activities as curcumin [22, 41]. Therefore, the protonated ions at $\mathrm{m} / \mathrm{z} 367,369,371$, and 373 were simultaneously monitored by a split SIR descriptor. The total ion chromatogram for the four ions monitored by SIR HPLC-MS for the curcumin reduction system is shown in Figure 4. It was observed that four chromatographic peaks were completely separated within less than 15 min under the gradient elution condition. However, the monitored ion at $\mathrm{m} / \mathrm{z}$ 365 was not detected under the above conditions. This might be caused by its extremely low trace level, which did not reach the detection sensitivity of ESI-MS. The chromatographic peak at the retention time of $0.79 \mathrm{~min}$ was the solvent peak. The corresponding mass spectra of chromatographic peaks at the retention time of 7.07, 8.62, and $12.72 \mathrm{~min}$ are shown in Figure 5. As shown in Figure 5a, c, and e, three paired ions with the mass difference of $2.00 \mathrm{Da}(\mathrm{m} / \mathrm{z} 371,373$, and $375 ; \mathrm{m} / \mathrm{z} 369$, 371 , and $373 ; m / z 367,369$, and 371) were, and their relative mass spectrometry signal intensities were similar to their parent compounds. We considered that $m / z 371(367+4 \mathrm{H}), \mathrm{m} / z 369$ $(367+2 \mathrm{H})$, and $\mathrm{m} / \mathrm{z} 367$ were tetrahydrocurcumin, dihydrocurcumin, and curcumin, separately. Their structures were further confirmed by MS/MS. An extra energy of $25 \mathrm{eV}$ was applied to the extracted ions $\mathrm{m} / \mathrm{z} 371,369$, and 367 to get the corresponding fragments. As shown in Figure 5b, the fragment peaks of $\mathrm{m} / \mathrm{z} 148.68$ and 217.05 indicated the existence of curcumin. The fragment peaks of $\mathrm{m} / \mathrm{z} 148.77$ and 218.88 indicated the existence of dihydrocurcumin (Figure $5 \mathrm{~d})$. The fragment peaks of $\mathrm{m} / \mathrm{z} 150.85$ and 218.92 indicated the existence of tetrahydrocurcumin (Figure 5f).

Based on the data we obtained, we propose that major metabolic pathways for curcumin were reduced to dihydrocurcumin and tetrahydrocurcumin by an endogenous reductase system in HLMs, and glucuronidated by UDPglucuronosyl transferases, as shown in Figure 6. Several researches have reported that curcumin could be metabolized via conjugation (glucuronidation and sulfation) and reduction [42]. However, only glucuronidation and reduction products were detected in our experiment. The sulfate conjugates were not detected, which was caused by the absence of sulfatase enzyme in the HLMs metabolic reaction system. Therefore, the metabolic results were nearly in accordance with the literature reported under our experimental conditions. The results of the study show that the stable isotopic labeling strategy is an efficient approach to find metabolite candidates by direct detecting signals derived from labeled and non-labeled parent compounds with a specified mass-shift, and it is nearly not influenced by the metabolic complexity. 

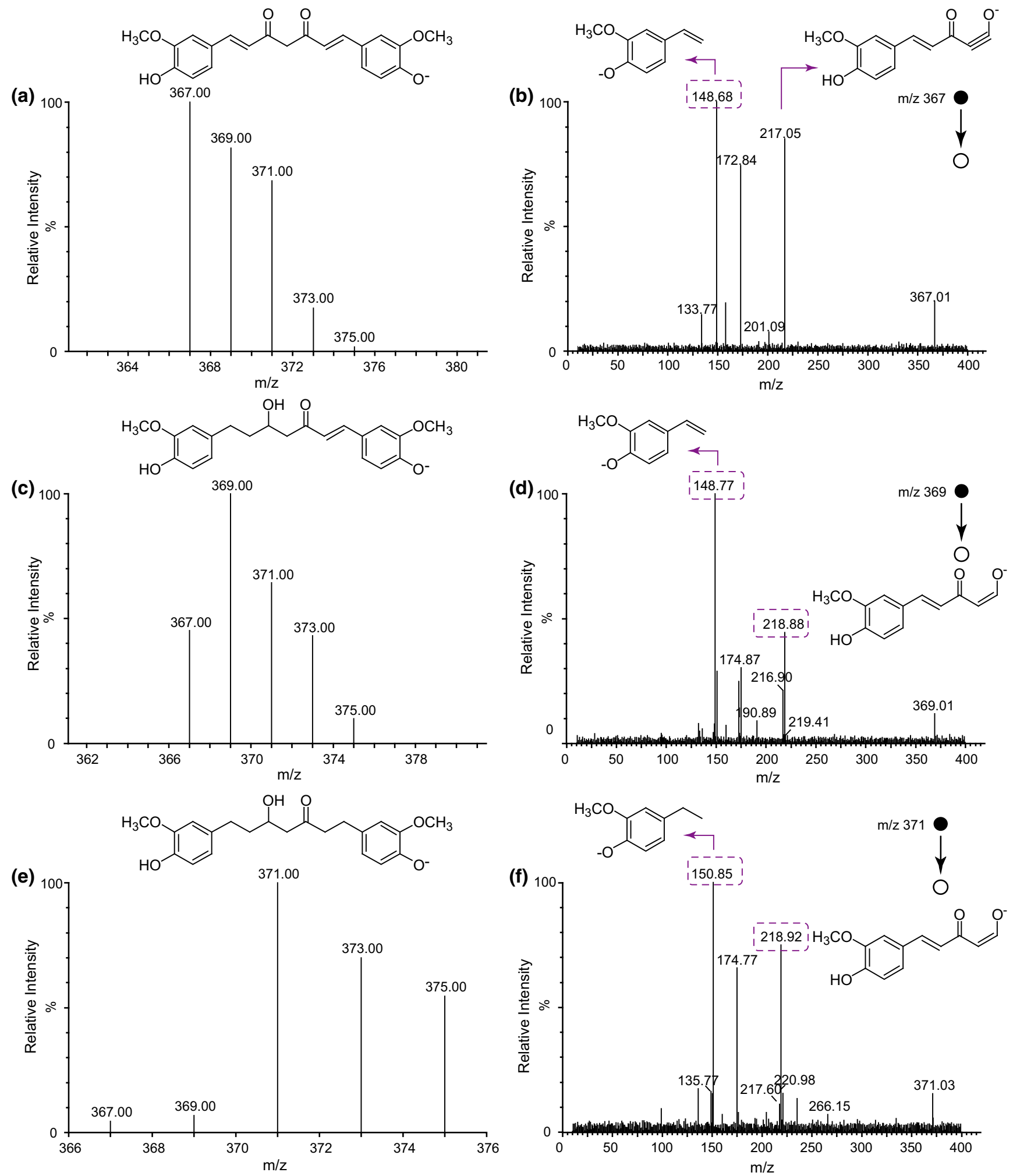

Figure 5. The corresponding mass spectra of the chromatographic peaks at the retention times of $7.07,8.62$, and $12.72 \mathrm{~min}$, and the corresponding tandem mass spectrometry. Spectra were obtained in the negative ion mode 


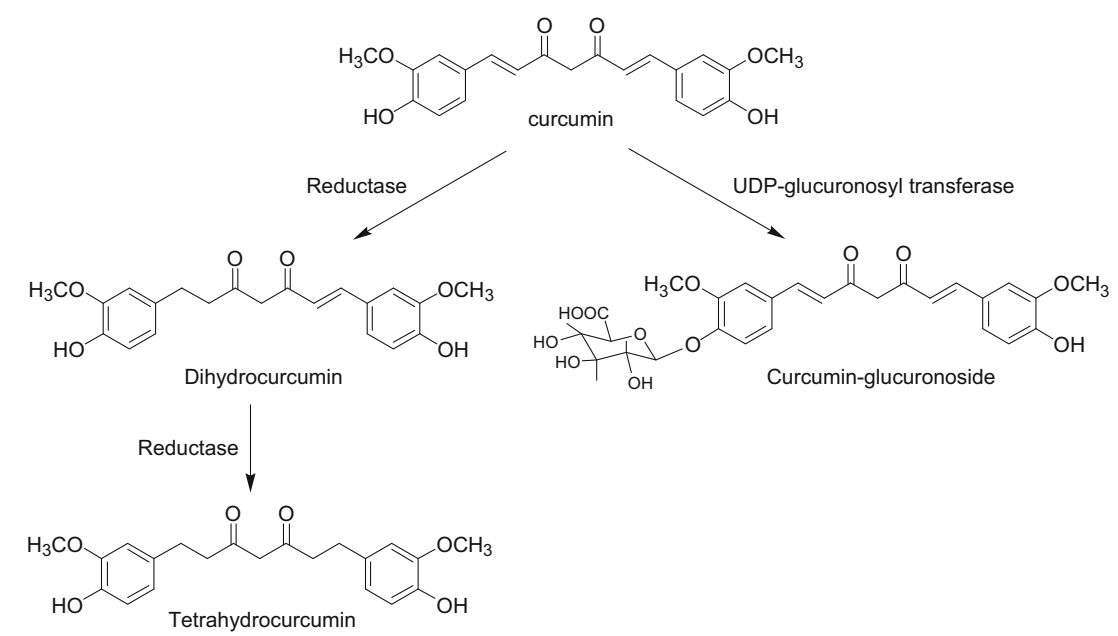

Figure 6. Proposed biotransformation and metabolites of curcumin in HLMs containing NADPH and UDPGA cofactors

\section{Conclusions}

We developed an isotopic labeling strategy for the discovery metabolites with Metabolynx software and the stable isotopic mass-shift at the similar retention time. The method is efficient and fast in detecting metabolite candidates even under a complex matrix. Curcumin was selected as a model compound to validate the feasibility of our approach. Three metabolites, including two reduction products and one glucuronide conjugate, were successfully detected under their specific HLMs metabolic conditions. The results indicate that the isotopic labeling approach together with the specific isotopic mass-shift and automatic data analysis has the ability to identify drug metabolites, which is very useful in drug discovery. In addition, if using high resolution MS for detection, the approach might become more accurate to filter a specific mass-shift.

\section{Acknowledgments}

The authors acknowledge support for this work by the National Youth Science Foundation (no. 21305074), the National Natural Science Foundation of China (no. 21172129 and no. 21475073), the National High Technology Research and Development Program of China (no. 2013AA092902), and China Postdoctoral Science Foundation (no. 2014M552238).

\section{References}

1. Hsieh, Y.S., Korfmacher, W.: The role of hyphenated chromatographymass spectrometry techniques in exploratory drug metabolism and pharmacokinetics. Curr. Pharm. Des. 15, 2251-2261 (2009)

2. Park, K.S., Kim, S.T., Kim, Y.M., Kim, Y., Lee, W.: The matrix effect of biological concomitant element on the signal intensity of Ge, As, and Se in inductively coupled plasma/mass spectrometry. Bull. Korean Chem. Soc. 23, 1389-1393 (2002)

3. Klein, S., Heinzle, E.: Isotope labeling experiments in metabolomics and fluxomics. Wiley Interdiscip. Rev. Syst. Biol. Med. 4, 261-272 (2012)
4. Chen, Q., Wu, J., Zhang, Y., Lin, J.M.: Qualitative and quantitative analysis of tumor cell metabolism via stable isotope labeling assisted microfluidic chip electrospray ionization mass spectrometry. Anal. Chem. 84, 1695$1701(2012)$

5. Avula, B., Tekwani, B.L., Chaurasiya, N.D., Nanayakkara, N.P.D., Wang, Y.H., Khan, S.I., Adelli, V.R., Sahu, R., Elsohly, M.A., McChesney, J.D., Khan, I.A., Walker, L.A.: Profiling primaquine metabolites in primary human hepatocytes using UHPLC-QTOF-MS with 13C stable isotope labeling. J. Mass Spectrom. 48, 276-285 (2013)

6. Liu, J., Tang, M.H., Lai, H.J., Dong, Y.F., Xie, C.F., Ye, H.Y., Ma, L., Qiu, N., Li, Y.F., Cai, L.L., Chen, L.J.: Identification of metabolites of honokiol in rat urine using $\mathrm{C}-13$ stable isotope labeling and liquid chromatography coupled with quadrupole time-of-flight tandem mass spectrometry. J. Chromatogr. A 1295, 48-56 (2013)

7. Uchida, M., Kanazawa, M., Ogiwara, A., Sezaki, H., Ando, A., Miyamoto, Y.: A computational drug metabolite detection using the stable isotopic mass-shift filtering with high resolution mass spectrometry in pioglitazone and flurbiprofen. Int. J. Mol. Sci. 14, 19716-19730 (2013)

8. Sano, M., Ferchaud-Roucher, V., Nael, C., Aguesse, A., Poupeau, G., Castellano, B., Darmaun, D.: Simultaneous detection of stable isotopelabeled and unlabeled L-tryptophan and of its main metabolites, Lkynurenine, serotonin and quinolinic acid, by gas chromatography/ negative ion chemical ionization mass spectrometry. J. Mass Spectrom. 49, 128-135 (2014)

9. Yang, W.C., Regnier, F.E., Jiang, Q., Adamec, J.: In vitro stable isotope labeling for discovery of novel metabolites by liquid chromatography-mass spectrometry: confirmation of gamma-tocopherol metabolism in human A549 cell. J. Chromatogr. A 1217, 667-675 (2010)

10. Lu, W.C., Sheen, J.F., Hwang, L.S., Wei, G.J.: Identification of $5,7,3^{\prime}, 4^{\prime}$-tetramethoxyflavone metabolites in rat urine by the isotopelabeling method and ultrahigh-performance liquid chromatographyelectrospray ionization-mass spectrometry. J. Agric. Food Chem. 60, 8123-8128 (2012)

11. Wei, G.J., Sheen, J.F., Lu, W.C., Hwang, L.S., Ho, C.T., Lin, C.I.: Identification of sinensetin metabolites in rat urine by an isotope-labeling method and ultrahigh-performance liquid chromatography-electrospray ionization mass spectrometry. J. Agric. Food Chem. 61, 5016-5021 (2013)

12. Ruseva, S., Lozanov, V., Markova, P., Girchev, R., Mitev, V.: In vivo investigation of homocysteine metabolism to polyamines by highresolution accurate mass spectrometry and stable isotope labeling. Anal. Biochem. 457, 38-47 (2014)

13. Baillie, T.A., Halpin, R.A., Matuszewski, B.K., Geer, L.A., Chavez-Eng, C.M., Dean, D., Braun, M., Doss, G., Jones, A., Marks, T., Melillo, D., Vyas, K.P.: Mechanistic studies on the reversible metabolism of rofecoxib to 5-hydroxyrofecoxib in the rat: Evidence for transient ring opening of a substituted 2-furanone derivative using stable isotope-labeling techniques. Drug Metab. Dispos. 29, 1614-1628 (2001)

14. Nelson, S.D., Trager, W.F.: The use of deuterium isotope effects to probe the active site properties, mechanism of cytochrome P450-catalyzed 
reactions, and mechanisms of metabolically dependent toxicity. Drug Metab. Dispos. 31, 1481-1498 (2003)

15. Yan, Z.Y., Caldwell, G.W.: Stable-isotope trapping and high-throughput screenings of reactive metabolites using the isotope MS signature. Anal. Chem. 76, 6835-6847 (2004)

16. He, K., Talaat, R.E., Woolf, T.F.: Incorporation of an oxygen from water into troglitazone quinone by cytochrome P450 and myeloperoxidase. Drug Metab. Dispos. 32, 442-446 (2004)

17. Daniels, J.S., Espina, R., Cao, K., Yuan, H.D., Lin, J.R., Diamond, S., Johnson, B., Rodgers, J., Prakash, S., Unger, S., Christ, D., Miwa, G., Gan, L.S., Mutlib, A.: Species-specific, P450-and sulfotransferase-mediated novel ring contraction of a naphthyridine$\mathrm{N}$-oxide compound in cynomolgus monkey. Chem. Res. Toxicol. 20, 1709-1717 (2007)

18. Holcapek, M., Kolarova, L., Nobilis, M.: High-performance liquid chromatography-tandem mass spectrometry in the identification and determination of phase I and phase II drug metabolites. Anal. Bioanal. Chem. 391, 59-78 (2008)

19. Aggarwal, B.B., Kumar, A., Bharti, A.C.: Anticancer potential of curcumin: preclinical and clinical studies. Anticancer Res. 23, 363-398 (2003)

20. Joe, B., Vijaykumar, M., Lokesh, B.R.: Biological properties of curcumincellular and molecular mechanisms of action. Crit. Rev. Food Sci. Nutr. 44, 97-111 (2004)

21. Rao, C.V., Rivenson, A., Simi, B., Reddy, B.S.: Chemoprevention of colon carcinogenesis by dietary curcumin, a naturally occurring plant phenolic compound. Cancer Res. 55, 259-266 (1995)

22. Garcea, G., Jones, D.J., Singh, R., Dennison, A.R., Farmer, P.B., Sharma, R.A., Steward, W.P., Gescher, A.J., Berry, D.P.: Detection of curcumin and its metabolites in hepatic tissue and portal blood of patients following oral administration. Br. J. Cancer 90, 1011-1015 (2004)

23. Maheshwari, R.K., Singh, A.K., Gaddipati, J., Srimal, R.C.: Multiple biological activities of curcumin: a short review. Life Sci. 78, 2081-2087 (2006)

24. Anand, P., Kunnumakkara, A.B..., Newman, R.A., Aggarwal, B.B.: Bioavailability of curcumin: problems and promises. Mol. Pharm. 4, 807-818 (2007)

25. Anand, P., Thomas, S.G., Kunnumakkara, A.B..., Sundaram, C., Harikumar, K.B., Sung, B., Tharakan, S.T., Misra, K., Priyadarsini, I.K., Rajasekharan, K.N., Aggarwal, B.B.: Biological activities of curcumin and its analogues (congeners) made by man and Mother Nature. Biochem. Pharmacol. 76, 1590-1611 (2008)

26. Cheng, A.L., Hsu, C.H., Lin, J.K., Hsu, M.M., Ho, Y.F., Shen, T.S., Ko, J.Y., Lin, J.T., Lin, B.R., Ming-Shiang, W., Yu, H.S., Jee, S.H., Chen, G.S., Chen, T.M., Chen, C.A., Lai, M.K., Pu, Y.S., Pan, M.H., Wang, Y.J., Tsai, C.C., Hsieh, C.Y.: Phase I clinical trial of curcumin, a chemopreventive agent, in patients with high-risk or pre-malignant lesions. Anticancer Res. 21, 2895-2900 (2001)
27. Hsu, C.H., Cheng, A.L.: Clinical studies with curcumin. Adv. Exp. Med. Biol. 595, 471-480 (2007)

28. Wahlang, B., Pawar, Y.B., Bansal, A.K.: Identification of permeabilityrelated hurdles in oral delivery of curcumin using the Caco-2 cell model. Eur. J. Pharm. Biopharm. 77, 275-282 (2011)

29. Shen, L., Ji, H.F.: The pharmacology of curcumin: is it the degradation products? Trends Mol. Med. 18, 138-144 (2012)

30. Ireson, C., Orr, S., Jones, D.J., Verschoyle, R., Lim, C.K., Luo, J.L., Howells, L., Plummer, S., Jukes, R., Williams, M., Steward, W.P., Gescher, A.: Characterization of metabolites of the chemopreventive agent curcumin in human and rat hepatocytes and in the rat in vivo, and evaluation of their ability to inhibit phorbol ester-induced prostaglandin E2 production. Cancer Res. 61, 1058-1064 (2001)

31. Shen, L., Ji, H.F.: Contribution of degradation products to the anticancer activity of curcumin. Clin. Cancer Res. 15, 7108. author reply 7108-7109 (2009)

32. Hoehle, S.I., Pfeiffer, E., Solyom, A.M., Metzler, M.: Metabolism of curcuminoids in tissue slices and subcellular fractions from rat liver. J. Agric. Food Chem. 54, 756-764 (2006)

33. Sharma, R.A., Steward, W.P., Gescher, A.J.: Pharmacokinetics and pharmacodynamics of curcumin. Adv. Exp. Med. Biol. 595, 453-470 (2007)

34. Cao, Y., Xu, R.X., Liu, Z.: A high-throughput quantification method of curcuminoids and curcumin metabolites in human plasma via highperformance liquid chromatography/tandem mass spectrometry. J. Chromatogr., B 949-950, 70-78 (2014)

35. King, C.D., Rios, G.R., Green, M.D., Tephly, T.R.: UDPglucuronosyltransferases. Curr. Drug Metab. 1, 143-161 (2000)

36. Dempe, J.S., Pfeiffer, E., Grimm, A.S., Metzler, M.: Metabolism of curcumin and induction of mitotic catastrophe in human cancer cells. Mol. Nutr. Food Res. 52, 1074-1081 (2008)

37. Guengerich, F.P.: Cytochrome p450 and chemical toxicology. Chem. Res. Toxicol. 21, 70-83 (2008)

38. Brandon, E.F., Raap, C.D., Meijerman, I., Beijnen, J.H., Schellens, J.H.: An update on in vitro test methods in human hepatic drug biotransformation research: pros and cons. Toxicol. Appl. Pharmacol. 189, 233-246 (2003)

39. Jia, L., Liu, X.: The conduct of drug metabolism studies considered good practice (II): in vitro experiments. Curr. Drug Metab. 8, 822-829 (2007)

40. Tamvakopoulos, C., Dimas, K., Sofianos, Z.D., Hatziantoniou, S., Han, Z., Liu, Z.L., Wyche, J.H., Pantazis, P.: Metabolism and anticancer activity of the curcumin analogue, dimethoxycurcumin. Clin. Cancer Res. 13, 12691277 (2007)

41. Marczylo, T.H., Steward, W.P., Gescher, A.J.: Rapid analysis of curcumin and curcumin metabolites in rat biomatrices using a novel ultraperformance liquid chromatography (UPLC) method. J. Agric. Food Chem. 57, 797-803 (2009)

42. Ireson, C.R., Jones, D.J., Orr, S., Coughtrie, M.W., Boocock, D.J., Williams, M.L., Farmer, P.B., Steward, W.P., Gescher, A.J.: Metabolism of the cancer chemopreventive agent curcumin in human and rat intestine. Cancer Epidemiol. Biomarkers Prev. 11, 105-111 (2002) 